\title{
LIN28B Polymorphisms Confer a Higher Postoperative Recurrence Risk in Reproductive-Age Women with Endometrial Polyps
}

\author{
Mei-Yin Lu $\mathbb{D},{ }^{1}$ Xiao-Hong Li $\mathbb{D}^{2},{ }^{2}$ Jia-Li Niu $\mathbb{D}^{1},{ }^{1}$ and Bin Liu $\mathbb{D}^{1}$ \\ ${ }^{1}$ Department of Biobank, Shenzhen Baoan Women's and Children's Hospital, Jinan University, Shenzhen, Guangdong, China \\ ${ }^{2}$ Department of Reproductive Health, Shenzhen Baoan Women's and Children's Hospital, Jinan University, Shenzhen, \\ Guangdong, China
}

Correspondence should be addressed to Bin Liu; gz12liubin@163.com

Received 13 December 2021; Revised 14 February 2022; Accepted 16 February 2022; Published 27 February 2022

Academic Editor: Dong Pan

Copyright (c) 2022 Mei-Yin Lu et al. This is an open access article distributed under the Creative Commons Attribution License, which permits unrestricted use, distribution, and reproduction in any medium, provided the original work is properly cited.

\begin{abstract}
The RNA-binding protein LIN28B is an important factor for cell proliferation. Because LIN28B polymorphisms have been shown to be relative with the recurrence of some hyperplastic diseases, we hypothesized that genetic variants of LIN28B gene were associated with postoperative recurrence risk in reproductive-age women with endometrial polyps (EP). In a hospital-based cohort of 351 reproductive female patients underwent hysteroscopic polypectomies between May 2018 and Jan 2020, we genotyped two common polymorphisms in LIN28B gene (rs369065 C > T and rs314280 A $>\mathrm{G}$ ) and analyzed their associations with the risk of postoperative recurrence in multiple Cox regression model. When followed up to Jun 2021, carries of rs369065 TT genotype had an increased risk of polyp recurrence (adjusting hazard ratio $[\mathrm{HR}]=1.883,95 \%$ confidence interval $[\mathrm{CI}]=1.033-3.434$ ) and had a shorter time to recurrence (median time 352 vs. 342 days, log-rank $P<0.01$ ), compared to the CC/CT genotype. Further stratification analysis showed that the increased risk of rs369065 TT genotype was more evident in patients who were older than 33 years (adjusted $\mathrm{HR}=2.597,95 \% \mathrm{CI}=1.037-6.505$ ), had a single polyp (adjusted $\mathrm{HR}=2.545,95 \% \mathrm{CI}=1.059-6.113$ ), and had smaller polyps $(<1.2 \mathrm{~cm}$, adjusted $\mathrm{HR}=2.708,95 \% \mathrm{CI}=1.042-7.043)$. However, no significant association between $\mathrm{rs} 314280 \mathrm{~A}>\mathrm{G}$ polymorphism and the risk of polyp recurrence was found. Our study suggests that rs369065 TT genotype of LIN28B gene is associated with an increased postoperative recurrence risk in EP patients, especially in those with fewer and smaller polyps. These findings implicate a precise choice of clinical counseling and decision making. Larger studies in different ethnic populations are warranted.
\end{abstract}

\section{Introduction}

Endometrial polyp (EP) is a focal hyperplasia of endometrial basal layer, composing of endometrial glands, vessels, and nearby stroma [1]. It is a common gynecologic disease with higher prevalence in infertile women [2]. Hysteroscopic polypectomy is commonly used to treat EP in infertile patients, which can improve spontaneous conception as well as assisted reproduction [2-4]. However, the rate of polyp recurrence after polypectomy remains $2.5 \sim 44 \%$, which hinders the improvement of fertility in these patients [2-5]. Therefore, postoperative recurrence of EP is now a concern for clinical counseling and decision making in reproductive-age women.
Limited reports to explore risk factors of polyp recurrence are available, mostly focused on postmenopausal women [1]. Only a few studies conducted in reproductiveage women have suggested some risk factors, such as the duration of follow-up, the number and size of polyps, and sex hormone therapy $[2-4,6]$. Most of the abovementioned risk factors are related to proliferation potential of endometrial cells. Thus, the biomarkers for hyperplasia of endometrial squamous cells should be further studied.

The highly conserved RNA-binding protein LIN28 plays an important role in cell proliferation [7]. Human produce two LIN28 paralogs, LIN28A and LIN28B, which have been shown to be genetically associated with many hyperplastic 
diseases [8, 9]. Compared to LIN28A, LIN28B is a key regulator of the proliferation of endometrial squamous cells [10]. Therefore, LIN28B gene was analyzed in this study. Human LIN28B gene (HGNC: 32207 ) spans $14.6 \mathrm{~kb}$ on chromosome 6q16.3-q21, contains seven exons, and encodes a 250aa RNA-binding protein LIN28B. LIN28B negatively regulates pre-miRNA processing (GO: 2000632), including let-7 family, miR107, miR-143, and miR-200c [11]. It binds these premiRNAs and sequesters them away from the microprocessor complex, hence prevents them mature [11]. The overexpression of LIN28B is seen in various primary tumors, linked to the repression of let-7 family of microRNAs and derepression of let-7 targets, which facilitates cell proliferation [12-14]. Additionally, there were adequate researches about the effect of LIN28B on tumor recurrence as well. The aberrant expression of LIN28B is related to the recurrence of Wilms tumor [15], hepatocellular carcinoma [16], colon cancer [17], ovarian cancer [18], adrenocortical cancer, and so on $[19,20]$. Moreover, LIN28B polymorphisms are significantly associated with recurrence of colorectal cancer [21]. However, no studies have reported the association between LIN28B polymorphisms and polyp recurrence after polypectomy. We hypothesized that genetic variants of LIN28B gene were associated with postoperative recurrence risk in EP patients.

In this hospital-based study conducted in a southern Chinese population between 2018 and 2021, we genotyped rs369065 C > T and rs314280 A > G polymorphisms in 351 reproductive female patients underwent hysteroscopic polypectomies and analyzed their associations with the risk of postoperative recurrence.

\section{Methods}

2.1. Study Population. This study is a genetic association study based on a hospital cohort, conducted in accordance with the reporting guidelines and checklist of Extension for Genetic Association Studies (STREGA) (Supplementary Table 1) [22]. In this study, $351 \mathrm{EP}$ patients aged 20 40 years routinely diagnosed by hysteroscopy, ultrasonic imaging, and pathological examinations were enrolled at the Department of Reproductive Health in Shenzhen Baoan Women's and Children's Hospital of Jinan University between May 2018 and Jan 2020. We excluded patients with previous polypectomy, or any form of diffused endometrial hyperplasia. To improve or reserve the fertility, all these EP patients have undergone hysteroscopic polypectomy under sedation or intradural anesthetic, following the routine procedure of our hospital.

As for follow-up, all enrolled patients were scheduled to come back for routine examinations every six months after polypectomy, up until Jun 2021. Because all these patients have the intention to future pregnancy, no loss to followup has been reported in this study. The recurrence status was verified by ultrasonic imaging and pathological examinations according to two criteria: the same type of histology and the same location, which differentiates it from a new polyp [5]. We collected the patients' information from their electronic medical records, including age, menarche age, menses, parity, gravidity, dysmenorrhea, abnormal uterine bleeding, endometritis, pelvic infection, salpingitis, endometriosis, intrauterine adhesion, and blood analysis before polypectomy, the number, size and location of polyps, endometrial thickness, type of hysteroscopic polypectomy, and follow-up duration (disease-free interval until symptomatic recurrence). $5 \mathrm{ml}$ of heparin anticoagulant blood of all enrolled patients was collected to perform genotyping. The participants signed a consent statement of ethical approval to use their samples and data.

This study was approved by the Ethics Committee of Shenzhen Baoan Women's and Children's Hospital, Jinan University (IRB No: LLSC-2018-08-01).

2.2. Single-Nucleotide Polymorphism Selection and Genotyping. We selected the single-nucleotide polymorphisms (SNPs) in LIN28B gene by using the dbSNP database according to the following three criteria [23]: (1) the minor allele frequencies (MAF) reported in HapMap database (http://hapmap.ncbi.nlm.nih.gov/) were more than $20 \%$ for Chinese Han subjects; (2) SNPs in low linkage disequilibrium with each other $\left(R^{2}<0.8\right)$. Based on these criteria, two SNPs rs369065 C > T and rs314280 A > G were selected in the present study.

Genomic DNA was extracted from blood samples using the MagaBio plus Blood DNA Kit (Bioer Technology, Hangzhou, China) according to the manufacturer's instructions. As described previously [23], genotyping of the abovementioned SNPs using Agenda Massarray technique was performed at CapitalBio Technology (Beijing, China), according to the manufacturer's protocol available at (doi:10.1007/9781-4939-6442-0_5). Briefly, the process involved a locusspecific PCR reaction and a single-base extension reaction. The PCR products were purified by resin and added into a SpectroCHIP (Agena Bioscience, Sequenom, San Diego, California, USA), then were analyzed by MALDI-TOF techniques. As shown in Supplementary Figure 1, the call of genotyping was conducted by TYPER 4.0 (SEQUNOM). Genotyping was repeated on a random $\sim 20 \%(70 / 351)$ of the samples, and the results were $100 \%$ concordant. All genotyping data of rs369065 C > T and rs314280 A > G polymorphisms were used for further analyses.

2.3. Statistical Analysis. To avoid bias of data collection, the data used for analyses were double-checked from the original medical records by two different research assistants. Missing data are very common in clinical researches, however, they were observed in the present study to a considerably low percentage ( $<5 \%$ of blood analyses and menstrual cycle/duration) and were replaced by the mean value of the variables. Chisquare test and Student's $t$ test were used to compare the differences between recurrent and nonrecurrent patients regarding demographic characteristics. The Chi-Square goodness of fit test was applied to indicate whether the genotype frequency distribution of each polymorphism in all subjects under study was in Hardy-Weinberg equilibrium. The polyp recurrence rate and median recurrence time between different genotype groups were analyzed by Kaplan-Meier Survival Curve, logrank test, and multiple Cox regression analysis by adjusting 
TABLE 1: Clinical and demographic characteristics of endometrial polyps patients with postoperative recurrence and nonrecurrence.

\begin{tabular}{|c|c|c|c|}
\hline Variables & Recurrence, $n(\%)$ & Nonrecurrence, $n(\%)$ & $P^{\mathrm{a}}$ \\
\hline All subjects & $44(100.0)$ & $307(100.0)$ & \\
\hline \multicolumn{4}{|l|}{ Age, years } \\
\hline$<33$ & $23(52.3)$ & $184(59.9)$ & \multirow[t]{2}{*}{0.352} \\
\hline$\geq 33$ & $21(47.7)$ & $123(40.1)$ & \\
\hline \multicolumn{4}{|l|}{ Menarche age (years) } \\
\hline$<13$ & $2(4.5)$ & $27(8.8)$ & \multirow[t]{2}{*}{0.506} \\
\hline$\geq 13$ & $42(95.5)$ & $280(91.2)$ & \\
\hline \multicolumn{4}{|l|}{ Menstrual cycle (days) } \\
\hline $23-35$ & $40(90.9)$ & $264(86.0)$ & \multirow[t]{2}{*}{0.305} \\
\hline$>35$ & $4(9.1)$ & $43(14.0)$ & \\
\hline \multicolumn{4}{|l|}{ Menstrual duration (days) } \\
\hline$\leq 7$ & $33(75.0)$ & $277(90.2)$ & \multirow[t]{2}{*}{0.934} \\
\hline$>7$ & $11(25.0)$ & $30(9.8)$ & \\
\hline Dysmenorrhea & $6(13.6)$ & $61(19.9)$ & 0.325 \\
\hline Abnormal uterine bleeding & $4(9.1)$ & $10(3.3)$ & 0.151 \\
\hline Endometritis & $16(36.4)$ & $101(32.8)$ & 0.648 \\
\hline Pelvic infection & $2(4.5)$ & $7(2.3)$ & 0.705 \\
\hline Salpingitis & $0(0.0)$ & $10(3.3)$ & 0.465 \\
\hline Endomotriosis & $1(2.3)$ & $5(1.6)$ & 0.754 \\
\hline Intrauterine adhesion & $1(2.3)$ & $7(2.3)$ & 0.591 \\
\hline \multicolumn{4}{|l|}{ Gravidities } \\
\hline 0 & $16(36.4)$ & $126(41.0)$ & \multirow[t]{3}{*}{0.470} \\
\hline 1 & $12(27.3)$ & $81(26.4)$ & \\
\hline$\geq 2$ & $16(36.4)$ & $100(32.6)$ & \\
\hline \multicolumn{4}{|l|}{ Abortion } \\
\hline 0 & $28(63.6)$ & $208(67.8)$ & \multirow[t]{3}{*}{0.610} \\
\hline 1 & $11(25.0)$ & $66(21.5)$ & \\
\hline$\geq 2$ & $5(11.4)$ & $33(10.8)$ & \\
\hline \multicolumn{4}{|l|}{ Deliveries } \\
\hline 0 & $20(45.5)$ & $164(53.4)$ & \multirow[t]{3}{*}{0.429} \\
\hline 1 & $15(34.1)$ & $82(26.7)$ & \\
\hline$\geq 2$ & $9(20.5)$ & $61(19.9)$ & \\
\hline \multicolumn{4}{|l|}{ Red blood cells $\left(\times 10^{12} / \mathrm{L}\right)$} \\
\hline$<4.37$ & $23(52.3)$ & $156(50.8)$ & \multirow[t]{2}{*}{0.602} \\
\hline$\geq 4.37$ & $21(47.7)$ & $151(49.2)$ & \\
\hline \multicolumn{4}{|l|}{ Hemoglobin $(\mathrm{g} / \mathrm{L})$} \\
\hline$<126$ & $28(63.64)$ & $146(47.56)$ & \multirow[t]{2}{*}{0.019} \\
\hline$\geq 126$ & $16(36.36)$ & $161(52.44)$ & \\
\hline \multicolumn{4}{|l|}{ White blood cells $\left(\times 10^{9} / \mathrm{L}\right)$} \\
\hline$<6.09$ & $25(56.82)$ & $150(48.86)$ & \multirow[t]{2}{*}{0.206} \\
\hline$\geq 6.09$ & $19(43.18)$ & $157(51.14)$ & \\
\hline \multicolumn{4}{|l|}{ Number of polyps } \\
\hline Single polyp & $21(47.73)$ & $196(63.84)$ & \multirow[t]{2}{*}{0.059} \\
\hline Multiple polyps & $23(52.27)$ & $111(36.16)$ & \\
\hline \multicolumn{4}{|l|}{ Diameter of polyps $(\mathrm{cm})$} \\
\hline$<1.2$ & $18(40.9)$ & $210(68.4)$ & 0.013 \\
\hline$\geq 1.2$ & $26(59.1)$ & $97(31.6)$ & \\
\hline
\end{tabular}


TABLE 1: Continued.

\begin{tabular}{lcc}
\hline Variables & Recurrence, $n(\%)$ & Nonrecurrence, $n(\%)$ \\
\hline Endometrial thickness $(\mathrm{cm})$ & & \\
$\quad<0.9$ & $22(50.0)$ & $153(49.2)$ \\
$\quad \geq 0.9$ & $22(50.0)$ & $154(50.8)$ \\
Average follow-up days & & $801.5 \pm 224.7$ \\
\hline
\end{tabular}

Notes: ${ }^{\mathrm{a} C h i}$-square tests or Fisher exact test.

TABLE 2: The associations between LIN28B gene polymorphisms and recurrence of EP.

\begin{tabular}{|c|c|c|c|c|c|}
\hline Genotypes & Recurrence, $n(\%)$ & Nonrecurrence, $n(\%)$ & $P$ for $\mathrm{HWP}^{\mathrm{a}}$ & Adjusted HR $(95 \% \mathrm{CI})^{\mathrm{b}}$ & $P$ for Cox model \\
\hline Subjects & 44 & 307 & & & \\
\hline \multicolumn{6}{|c|}{ rs369065 $(\mathrm{C}>\mathrm{T})$} \\
\hline $\mathrm{CC}$ & $5(11.4)$ & $48(15.6)$ & 0.576 & Ref. (1.00) & \\
\hline CT & $16(36.4)$ & $160(51.1)$ & & $1.115(0.406-3.061)$ & 0.833 \\
\hline $\mathrm{TT}$ & $23(52.3)$ & $99(32.2)$ & & $2.044(0.768-5.442)$ & 0.152 \\
\hline Dominant & $39(88.6)$ & $259(84.4)$ & & $1.512(0.591-3.867)$ & 0.388 \\
\hline Recessive & $21(47.7)$ & $208(67.8)$ & & $1.883(1.033-3.434)$ & 0.039 \\
\hline \multicolumn{6}{|c|}{ rs314280 $(\mathrm{A}>\mathrm{G})$} \\
\hline AA & $3(6.8)$ & $30(9.8)$ & 0.974 & Ref. (1.00) & \\
\hline GA & $13(29.5)$ & $138(45.0)$ & & $0.904(0.249-3.275)$ & 0.878 \\
\hline GG & $28(63.6)$ & $139(45.3)$ & & $1.613(0.472-5.5 .7)$ & 0.446 \\
\hline Dominant & $41(93.2)$ & $277(90.2)$ & & $1.271(0.382-4.234)$ & 0.696 \\
\hline Recessive & $16(36.4)$ & $168(54.7)$ & & $1.751(0.916-3.349)$ & 0.090 \\
\hline
\end{tabular}

aThe observed genotype frequency in the study population was in agreement with the Hardy-Weinberg equilibrium $\left(p^{2}+2 \mathrm{pq}+p^{2}=1\right)\left(\chi^{2}=1.103, P=0.576\right.$ for rs369065; $\chi^{2}=0.053, P=0.974$ for rs314280). HR: hazard ratio. ${ }^{\mathrm{b}}$ Adjusted age, gravidities, menarche age, number and size of polyps, and endometrial thickness.

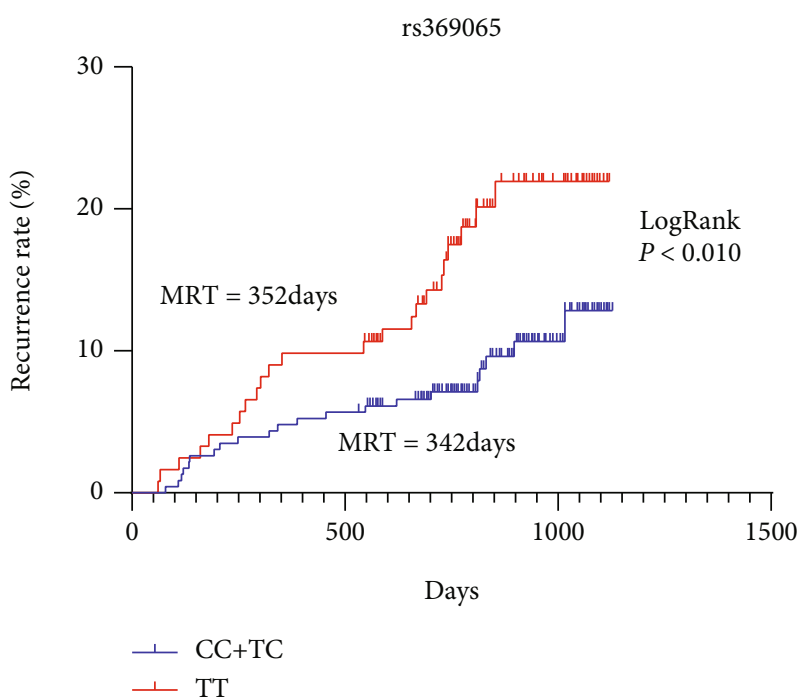

Figure 1: Kaplan-Meier survival curve for polyp recurrence by LIN28B rs369065 C > T polymorphism.

for age, gravidities, menarche age, the number and size of polyps, and endometrial thickness. The cut-off values for the abovementioned confounding factors were determined by using X-tile v3.6.1 (Yale University) [24]. Further stratification analyses by age, gravidities, circulating hemoglobin level, the number and size of polyps, and endometrial thickness were performed as well. The study size and statistical power were calculated using the PASS 15.0 software (NCSS LLC., Kaysville, Utah, USA). And the false-positive report probability (FPRP) test was applied to detect the false-positive association findings. All statistical analyses were performed using SPSS 18.0 software (IBM, Armonk, NY, USA). A two-sided statistical significance level of 0.05 was chosen as well.

\section{Results}

3.1. Characteristics of the Study Population. The average follow-up time was $801.5 \pm 224.7$ days. Until Jun 2021, there were 44 patients recurrent (12.5\%). The distribution of demographic characteristics of patients is shown in Table 1. Overall, the differences in distributions of age, menses, parity, gravidity, dysmenorrhea, abnormal uterine bleeding, endometritis, pelvic infection, salpingitis, endometriosis, intrauterine adhesion, polyps' number, and endometrial thickness between the recurrent and nonrecurrent patients were not statistically significant (all $P$ values $>0.05$ ). However, the recurrent patients were more probably to have a lower level of circulating hemoglobin and have bigger polyps than were nonrecurrent patients $(P$ values were 0.019 and 0.013 , respectively) (Table 1$)$. Therefore, these two variables, 
TABLE 3: Stratification analysis of LIN28B rs369065 polymorphism by selected variables in recurrent and nonrecurrent patients.

\begin{tabular}{|c|c|c|c|c|c|c|}
\hline & Recurre & $(n=44)$ & Nonrecurr & $(n=307)$ & & \\
\hline & $\begin{array}{c}\text { CC/TC } \\
n(\%)\end{array}$ & $\begin{array}{c}\mathrm{TT} \\
n(\%)\end{array}$ & $\begin{array}{c}\text { CC/CT } \\
n(\%)\end{array}$ & $\begin{array}{c}\text { TT } \\
n(\%)\end{array}$ & Adjusted HR $(95 \% \text { CI })^{\mathrm{a}}$ & $P$ \\
\hline Age (years) & & & & & & \\
\hline$<33$ & $13(56.5)$ & $10(43.5)$ & $121(65.8)$ & $63(34.2)$ & $1.300(0.559-3.022)$ & 0.542 \\
\hline$\geq 33$ & $8(38.1)$ & $13(61.9)$ & $87(70.7)$ & $36(29.3)$ & $2.597(1.037-6.505)$ & 0.042 \\
\hline Gravidities & & & & & & \\
\hline 0 & $7(43.8)$ & $9(56.2)$ & $78(60.9)$ & $48(39.1)$ & $1.565(0.570-4.299)$ & 0.385 \\
\hline 1 & $6(50.0)$ & $6(50.0)$ & $61(75.3)$ & $20(24.7)$ & $1.886(0.556-6.402)$ & 0.309 \\
\hline$\geq 2$ & $8(50.0)$ & $8(50.0)$ & $69(69.0)$ & $31(31.0)$ & $1.914(0.708-5.173)$ & 0.201 \\
\hline Hemoglobin $(\mathrm{g} / \mathrm{L})$ & & & & & & \\
\hline$<126$ & $14(31.82)$ & $14(31.82)$ & $95(30.94)$ & $51(16.61)$ & $1.837(0.850-3.973)$ & 0.122 \\
\hline$\geq 126$ & $7(15.91)$ & $9(20.45)$ & $113(36.81)$ & $48(15.64)$ & $1.862(0.657-5.275)$ & 0.242 \\
\hline Number of polyps & & & & & & \\
\hline Single polyp & $10(47.6)$ & $11(52.4)$ & $139(70.9)$ & $57(29.1)$ & $2.545(1.059-6.113)$ & 0.037 \\
\hline Multiple polyps & $11(47.8)$ & $12(52.2)$ & $69(62.2)$ & $42(37.8)$ & $1.570(0.683-3.611)$ & 0.288 \\
\hline Diameter of polyp & & & & & & \\
\hline$<1.2$ & $18(47.4)$ & $20(52.6)$ & $170(69.4)$ & $85(30.6)$ & $2.708(1.042-7.043)$ & 0.041 \\
\hline$\geq 1.2$ & $3(50.0)$ & $3(50.0)$ & $38(73.1)$ & $14(26.9)$ & $1.458(0.672-3.165)$ & 0.340 \\
\hline Endometrial thickr & & & & & & \\
\hline$<0.9$ & $10(45.5)$ & $12(54.5)$ & $102(66.7)$ & $51(33.3)$ & $2.189(0.920-5.209)$ & 0.077 \\
\hline$\geq 0.9$ & $11(50.0)$ & $11(50.0)$ & $106(68.8)$ & $48(31.2)$ & $1.774(0.750-4.200)$ & 0.192 \\
\hline
\end{tabular}

HR: hazard ratio. ${ }^{2}$ Adjusted age, gravidities, menarche age, number and size of polyps, and endometrial thickness.

combined with other potential risk factors for polyp recurrence, were further adjusted for in the multivariate Cox regression model to control possible confounding on the main effects of the study polymorphisms. Additionally, they were used in later stratification analysis.

3.2. Association of LIN28B Polymorphisms with Postoperative Recurrence Risk in EP. The genotype distributions of the LIN28B rs369065 C > T and rs314280 A > G polymorphisms among the recurrent and non-recurrent patients are summarized in Table 2. The observed genotype frequencies of these two polymorphisms were all in agreement with the HardyWeinberg equilibrium in the study population ( $P$ values were 0.576 and 0.974 , respectively). To control the statistical bias, we adjusted confounding factors in multiple Cox model using age, gravidities, menarche age, the size and number of polyps, and endometrial thickness.

As shown in Table 2, rs369065 C > T polymorphism was significantly associated with recurrence risk in a recessive genetic model: compared to the rs369065 CC/CT genotypes, carriers of TT genotype had an increased risk of polyp recurrence (adjusted hazard ratio $[\mathrm{HR}]=1.883,95 \%$ confidence interval $[\mathrm{CI}]=1.033-3.434, P=0.039)$. However, for the rs314280 A > G polymorphism, there was no significant association between this polymorphism and recurrence risk in either of genetic models.

Further Kaplan-Meier survival curve and log-rank test showed that carries of rs369065 TT genotype had a shorter time to recurrence, compared with the CC/CT genotype (median time 352 vs. 342 days, $\log$-rank $P<0.01$ ) (Figure 1 ).
3.3. Stratification Analysis of LIN28B rs369065 TT Genotype and Risk of Polyp Recurrence. We further performed a stratification analysis of the associations between LIN28B rs369065 TT genotypes and risk of polyp recurrence by subgroups of age, gravidities, circulating hemoglobin level, the number and size of polyps, and endometrial thickness. As shown in Table 3, the increased risks of polyp recurrence associated with the rs369065 TT genotypes did not differ by gravidities, circulating hemoglobin level, and endometrial thickness $(P>0.05$ for all subgroups). However, the harmful role of rs369065 TT genotypes in recurrence risk was more evident in the subjects with an older age ( $>33$ years, adjusted $\mathrm{HR}=2.597, \quad 95 \% \mathrm{CI}=1.037-6.505, \quad P=0.042)$, a single polyp (adjusted $\mathrm{HR}=2.545,95 \% \mathrm{CI}=1.059-6.113, P=$ $0.037)$, and a smaller polyp $(<1.2 \mathrm{~cm}$, adjusted $\mathrm{HR}=2.708$, $95 \% \mathrm{CI}=1.042-7.043, P=0.041)$. For the $\operatorname{rs} 314280 \mathrm{~A}>\mathrm{G}$ polymorphism, no significant association was found in any subgroup (data not shown).

\section{Discussion}

In this study, we found that rs369065 TT genotype of LIN28B gene was associated with an increased postoperative recurrence risk of EP in reproductive-age women, and the deleterious role of rs369065 TT genotype in recurrence risk was more evident in the subgroups older than 33 years, and those with fewer and smaller polyps.

Although LIN28B is a key factor for the etiology of occurrence $[12,25,26]$ and recurrence $[15,18,20]$ for many hyperplastic diseases, no literature has analyzed its role in 


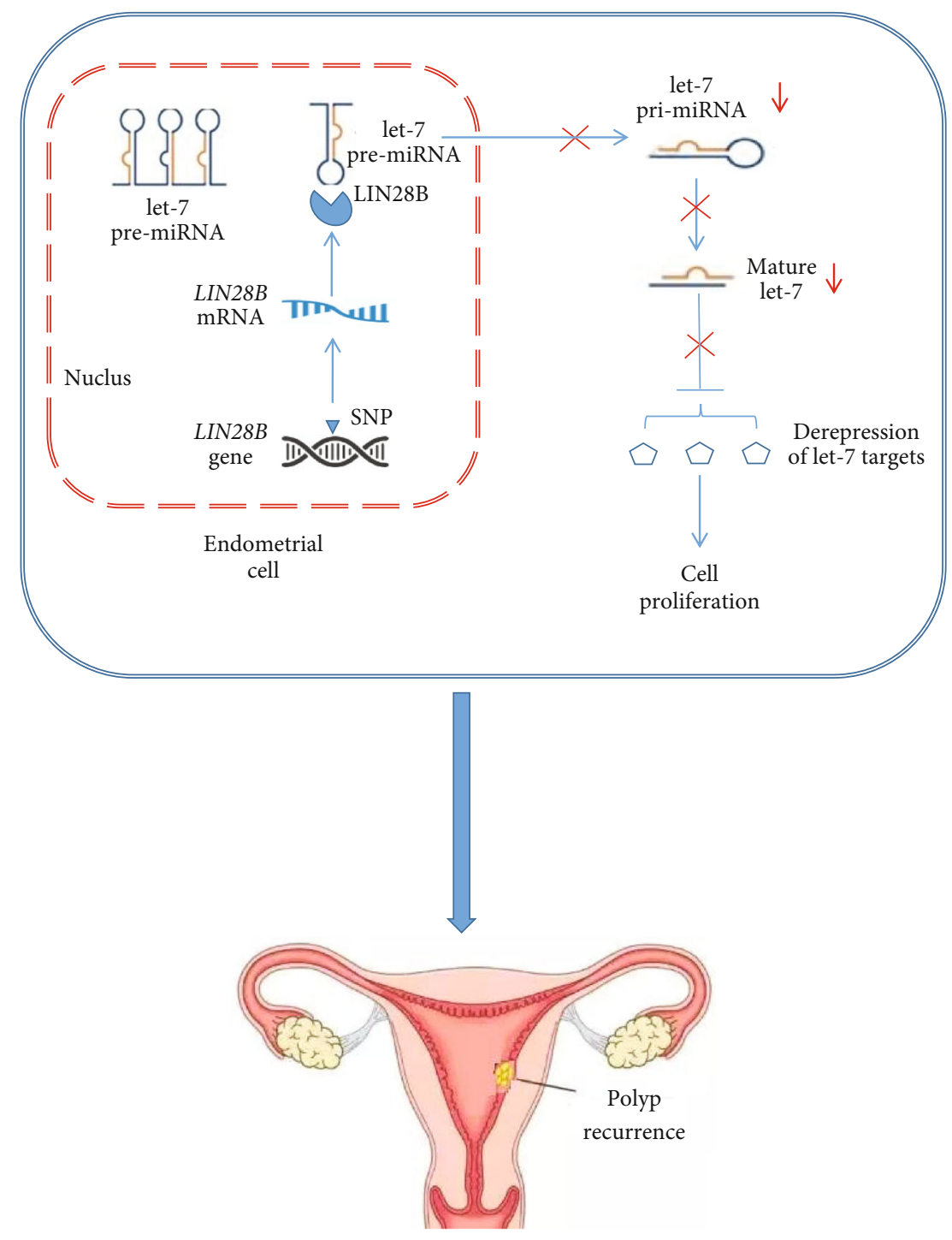

FIGURE 2: Potential mechanism of LIN28B polymorphisms in the recurrence of endometrial polyps.

the occurrence and recurrence of EP yet to our knowledge. In one of our previous studies conducted in $351 \mathrm{EP}$ patients and $493 \mathrm{EP}$-free controls in a reproductive women population in South China, we did not find significant associations between LIN28B polymorphisms and the occurrence of EP (data not reported). However, we found that LIN28B rs $369065 \mathrm{C}>\mathrm{T}$ polymorphism was significantly associated with an increased risk of polyp recurrence in this study. Through LIN28b/let-7 axis, LIN28B gene regulates the cell proliferation of endometrial stroma [10,13, 27-29], which is tightly linked with the recurrence of EP. Taken together with our data and above reports, our findings on the association between $L I N 28 B$ polymorphisms and polyp recurrence are plausible.

A few population-based studies have analyzed the association of LIN28B polymorphisms with various phenotypes. They showed that $L I N 28 B$ rs $369065 \mathrm{C}>\mathrm{T}$ polymorphism is significantly associated with chronic hepatitis $\mathrm{B}$ virus infection [30], standing height [31], and age at menarche [32,33]. These reports implied that $L I N 28 B$ rs $369065 \mathrm{C}>\mathrm{T}$ might be a potential functional polymorphism, which can alter LIN28B gene's expression or function, then promote the proliferation of endometrial squamous cells, thus, risk of polyp recurrence. However, there is another possible explanation: LIN28B rs $369065 \mathrm{C}>\mathrm{T}$ polymorphism is tightly linked with another functional polymorphism rs221634 $\mathrm{T}>\mathrm{A}$ in Chinese $\left(R^{2}>0.9\right)$. The substitution $\mathrm{T}>\mathrm{A}$ of $\mathrm{rs} 221634$ causes losing a binding site of hsa-mir-548 family in the $3^{\prime}$ UTR of human LIN28B gene, as analyzed by SNPinfo (https://manticore.niehs .nih.gov/). The hsa-mir-548 family is highly expressed in human reproductive tissues [34] and is a pivotal suppressor of cell growth [35-37]. So we can also explain our results by that $\mathrm{rs} 221634 \mathrm{~T}>\mathrm{A}$ polymorphism is the real genetic cause of polyp recurrence. Combined with the abovementioned analyses, our findings on the association between LIN28B polymorphisms and polyp recurrence are biological creditable. As shown in Figure 2, the polymorphisms of LIN28B gene will affect the gene's expression and then affect the binding of 
LIN28B with let-7 pre-miRNA, preventing them mature, and causing derepression of let-7 targets, thus risk of polyp recurrence.

In the subgroup analysis, we found that the deleterious role of rs369065 TT genotype was absent in the patients with more and bigger polyps, who are known as susceptible population for polyp recurrence $[3,38]$. The size and number of polyps relate to the cell proliferation of endometrial squamous cells, which is regulated by LIN28B/let-7 pathway [10]. This implies that the contribution of $\mathrm{rs} 369065 \mathrm{C}>\mathrm{T}$ polymorphism to polyp recurrence could be modulated by environmental risk factors. In contrast, patients with fewer and smaller polyps, but carrying the harmful rs369065 TT genotype, still have an increased recurrence risk in this study. These findings suggest that a more precise choice should be made in clinical counseling and decision making for EP treatment.

This study had some limitations because it was a hospital-based cohort study, restricted to a Chinese Han population. However, the genotype frequencies of the two study polymorphisms among our subjects well fit the Hardy-Weinberg disequilibrium law, and T allele frequency of rs369065 in our study (61.1\%) is similar to the frequencies reported in two studies conducted in two Chinese populations (64\% [30] and 67\% [39]), suggesting the subjects' selection is random. Additionally, we had achieved a more than 99\% study power (two-sided test, $\alpha=0.05$ ) to detect a HR of 1.883 for the rs369065 TT genotype (which occurred at a frequency of $32.2 \%$ in the nonrecurrence subjects) compared with the rs369065 CC/CT genotypes in a population of more than 300 subjects. Therefore, it appears that our finding that the rs369065 TT genotype associated with an increased risk of polyp recurrence is unlikely to have been by chance. Also, when we performed the FPRP analysis and found that under the assumption of a prior probability of 0.05 and a prior odds ratio of 1.5 as suggested by Wacholder et al. [40], the FPRP for the observed association between the rs369065 $\mathrm{C}>\mathrm{T}$ polymorphism and risk of polyp recurrence yielded a value of 0.165 , which was lower than the pre-set FPRP-level criterion 0.20, suggesting that this finding is noteworthy.

\section{Conclusions}

For the first time, we found that $L I N 28 B$ rs369065 C > T polymorphism was associated with an increased postoperative recurrence risk in reproductive-age women with EP, especially in some "low-risk" subpopulations, who had fewer and smaller polyps, implying a more precise choice of clinical counseling and decision making for EP patients. Larger studies in different ethnic populations are warranted.

\section{Data Availability}

The raw data supporting the conclusions of this article will be made available by the authors, without undue reservation.

\section{Ethical Approval}

The studies involving human participants were reviewed and approved by the Shenzhen Baoan Women's and Children's Hospital, Jinan University (IRB No: LLSC-2018-08-01).

\section{Consent}

The patients/participants provided their written informed consent to participate in this study.

\section{Conflicts of Interest}

The authors declare that there are no conflicts of interest regarding the publication of this paper.

\section{Authors' Contributions}

M-YL did the sample and data collection, follow-up, data analysis, graphs plotting, and manuscript drafting. X-HL did the project management, sample collection, follow-up, and result discussion. J-LN did the data double-check from the original medical records, data analysis, and result discussion. BL did the project design and management, experimental supervision, data analysis and discussion, and manuscript drafting and revision. All authors contributed to the article and approved the submitted version.

\section{Acknowledgments}

This work was supported by Research Foundation of Shenzhen Baoan Women's and Children's Hospital, Jinan University to BL (BAFY 2020005).

\section{Supplementary Materials}

Supplementary 1. Supplementary Figure 1: Cartesian plots of rs369065 C > T and rs314280 A > G polymorphisms analyzed using Agenda MassArray technique.

Supplementary 2. Supplementary Table 1: STREGA checklist for this study.

\section{References}

[1] S. Molnar, Z. Farkas, A. Jakab, R. Lampe, and P. Torok, "Effectiveness of different methods for polypectomy in the menopause: a retrospective study," Climacteric, vol. 23 , no. 4, pp. 325-329, 2020.

[2] O. Ceci, M. Franchini, S. Cardinale et al., "Comparison of endometrial polyp recurrence in fertile women after office hysteroscopic endometrial polypectomy using two widely spread techniques," The Journal of Obstetrics and Gynaecology Research, vol. 46, no. 10, pp. 2084-2091, 2020.

[3] F. Gu, H. Zhang, S. Ruan et al., "High number of endometrial polyps is a strong predictor of recurrence: findings of a prospective cohort study in reproductive-age women," Fertility and Sterility, vol. 109, no. 3, pp. 493-500, 2018.

[4] A. Ciscato, S. Y. Zare, and O. Fadare, "The significance of recurrence in endometrial polyps: a clinicopathologic analysis," Human Pathology, vol. 100, pp. 38-44, 2020. 
[5] J. Cea Garcia, A. Jimenez Caraballo, M. D. M. Rios Vallejo, and I. Zapardiel, "Retrospective cohort study on the symptomatic recurrence pattern after hysteroscopic polypectomy," Gynecology and Minimally Invasive Therapy, vol. 9, no. 4, pp. 209-214, 2020.

[6] Y. Wang, M. Yang, X. Huang, X. Li, E. Lin, and Y. Feng, "Prevention of benign endometrial polyp recurrence using a levonorgestrel- releasing intrauterine system in premenopausal patients: a retrospective cohort study," Journal of Minimally Invasive Gynecology, vol. 27, no. 6, pp. 1281-1286, 2020.

[7] M. Farzaneh, F. Attari, and S. E. Khoshnam, “Concise review: LIN28/let-7 signaling, a critical double-negative feedback loop during pluripotency, reprogramming, and tumorigenicity," Cellular Reprogramming, vol. 19, no. 5, pp. 289-293, 2017.

[8] H. Guo, Y. Liao, A. Lin et al., "Association betweenLIN28Agene polymorphisms and glioma susceptibility in Chinese children," Cancer Control, vol. 28, p. 107327482110400, 2021.

[9] Z. Yang, Y. Deng, K. Zhang et al., "LIN28Apolymorphisms and hepatoblastoma susceptibility in Chinese children," Journal of Cancer, vol. 12, no. 5, pp. 1373-1378, 2021.

[10] H. Li, D. Liu, L. Liu, S. Huang, A. Ma, and X. Zhang, "The role of HOTAIR/miR-152-3p/LIN28B in regulating the progression of endometrial squamous carcinoma," Archives of Medical Science, vol. 17, no. 2, pp. 434-448, 2021.

[11] S. I. Rangel-Guerrero, P. A. Franco-Urquijo, E. Martinez-Salas, and L. M. Alvarez-Salas, "Structural insights of the pre-let-7 interaction with LIN28B," Nucleosides, Nucleotides \& Nucleic Acids, vol. 40, no. 2, pp. 194-211, 2020.

[12] J. Lovnicki, Y. Gan, T. Feng et al., "LIN28B promotes the development of neuroendocrine prostate cancer," The Journal of Clinical Investigation, vol. 130, no. 10, pp. 5338-5348, 2020.

[13] J. Huang, D. Cao, J. Sha, X. Zhu, and S. Han, "DLL3 is regulated by LIN28B and miR-518d-5p and regulates cell proliferation, migration and chemotherapy response in advanced small cell lung cancer," Biochemical and Biophysical Research Communications, vol. 514, no. 3, pp. 853-860, 2019.

[14] S. L. Lin, H. Duan, S. Wang, and J. J. Li, "Overexpression of Lin28B promoted the proliferation of adenomyotic smooth muscle cells of the junctional zone via regulating Let-7a," Reproductive Sciences, vol. 27, no. 5, pp. 1156-1163, 2020.

[15] S. Gadd, V. Huff, A. L. Walz et al., "A children's oncology group and TARGET initiative exploring the genetic landscape of Wilms tumor," Nature Genetics, vol. 49, no. 10, pp. 14871494, 2017.

[16] S. W. Cheng, H. W. Tsai, Y. J. Lin et al., "Lin28B is an oncofetal circulating cancer stem cell-like marker associated with recurrence of hepatocellular carcinoma," PLoS One, vol. 8, no. 11, article e80053, 2013.

[17] M. Pang, G. Wu, X. Hou et al., "LIN28B promotes colon cancer migration and recurrence," PLoS One, vol. 9, no. 10, article e109169, 2014.

[18] Y. Li, J. Wang, F. Wang, W. Chen, C. Gao, and J. Wang, "RNF144A suppresses ovarian cancer stem cell properties and tumor progression through regulation of LIN28B degradation via the ubiquitin-proteasome pathway," Cell Biology and Toxicology, 2021.

[19] A. M. Faria, S. Sbiera, T. C. Ribeiro et al., "Expression of LIN28 and its regulatory microRNAs in adult adrenocortical cancer," Clinical Endocrinology, vol. 82, no. 4, pp. 481-488, 2015.

[20] J. Zhang, A. Xu, C. Miao, J. Yang, M. Gu, and N. Song, "Prognostic value of Lin28A and Lin28B in various human malignancies: a systematic review and meta-analysis," Cancer Cell International, vol. 19, no. 1, p. 79, 2019.

[21] Y. Ye, B. Madison, X. Wu, and A. K. Rustgi, "A LIN28B polymorphism predicts for colon cancer survival," Cancer Biology \& Therapy, vol. 13, no. 14, pp. 1390-1395, 2012.

[22] J. Little, J. P. Higgins, J. P. Ioannidis et al., "Strengthening the reporting of genetic association studies (STREGA): an extension of the STROBE statement," Annals of Internal Medicine, vol. 150, no. 3, pp. 206-215, 2009.

[23] Z. Q. Liu, M. Y. Lu, and B. Liu, "Polymorphisms in XPC gene and risk of uterine leiomyoma in reproductive women," Pathology Oncology Research, vol. 26, no. 3, pp. 1459-1464, 2020.

[24] S. Zhao, X. Chen, D. Wen, C. Zhang, and X. Wang, "Oncologic nomogram for stage I rectal cancer to assist patient selection for adjuvant (chemo)radiotherapy following local excision," Frontiers in Oncology, vol. 11, article 632085, 2021.

[25] W. Ji, Y. L. Diao, Y. R. Qiu, J. Ge, X. C. Cao, and Y. Yu, "LINC00665 promotes breast cancer progression through regulation of the miR-379-5p/LIN28B axis," Cell Death \& Disease, vol. 11, no. 1, p. 16, 2020.

[26] T. Tao, H. Shi, L. Mariani et al., "LIN28B regulates transcription and potentiates MYCN-induced neuroblastoma through binding to ZNF143 at target gene promotors," Proceedings of the National Academy of Sciences of the United States of America, vol. 117, no. 28, pp. 16516-16526, 2020.

[27] X. Zhang, M. Li, G. Sun, Y. Bai, D. Lv, and C. Liu, "MiR-563 restrains cell proliferation via targeting LIN28B in human lung cancer," Thorac Cancer, vol. 11, no. 1, pp. 55-61, 2020.

[28] J. Canfield, S. Arlier, E. F. Mong et al., "Decreased LIN28B in preeclampsia impairs human trophoblast differentiation and migration," The FASEB Journal, vol. 33, no. 2, pp. 2759$2769,2019$.

[29] J. Wu, X. Feng, Y. Du et al., " $\beta$-catenin/LIN28B promotes the proliferation of human choriocarcinoma cells via Let-7a repression," Acta Biochimica et Biophysica Sinica, vol. 51, no. 5, pp. 455-462, 2019.

[30] Q. Han, J. Sang, X. Fan et al., "Association of LIN28B polymorphisms with chronic hepatitis B virus infection," Virology Journal, vol. 17, no. 1, p. 81, 2020.

[31] C. F. Chabris, J. J. Lee, D. J. Benjamin et al., "Why it is hard to find genes associated with social science traits: theoretical and empirical considerations," American Journal of Public Health, vol. 103, Suppl 1, pp. S152-S166, 2013.

[32] K. L. Spencer, J. Malinowski, C. L. Carty et al., "Genetic variation and reproductive timing: African American women from the Population Architecture using Genomics and Epidemiology (PAGE) Study," PLoS One, vol. 8, no. 2, article e55258, 2013.

[33] C. T. Chen, L. Fernandez-Rhodes, R. G. Brzyski et al., "Replication of loci influencing ages at menarche and menopause in Hispanic women: the Women's Health Initiative SHARe Study," Human Molecular Genetics, vol. 21, no. 6, pp. 14191432, 2012.

[34] I. Rooda, B. Kaselt, M. Liivrand, O. P. Smolander, A. Salumets, and A. Velthut-Meikas, "Hsa-mir-548 family expression in human reproductive tissues," BMC Genomic Data, vol. 22, no. 1, p. 40, 2021.

[35] M. Saffari, S. M. H. Ghaderian, M. D. Omrani, M. Afsharpad, K. Shankaie, and N. Samadaian, "The association of miR-let 7b and miR-548 with PTEN in prostate cancer," Urology Journal, vol. 16, no. 3, pp. 267-273, 2019. 
[36] Z. Wang, X. Wu, X. Hou et al., "miR-548b-3p functions as a tumor suppressor in lung cancer," Lasers in Medical Science, vol. 35, no. 4, pp. 833-839, 2020.

[37] Y. Shi, M. Qiu, Y. Wu, and L. Hai, "MiR-548-3p functions as an anti-oncogenic regulator in breast cancer," Biomedicine \& Pharmacotherapy, vol. 75, pp. 111-116, 2015.

[38] V. Tanos, K. E. Berry, J. Seikkula et al., "The management of polyps in female reproductive organs," International Journal of Surgery, vol. 43, pp. 7-16, 2017.

[39] C. He, P. Kraft, C. Chen et al., "Genome-wide association studies identify loci associated with age at menarche and age at natural menopause," Nature Genetics, vol. 41, no. 6, pp. 724-728, 2009.

[40] S. Wacholder, S. Chanock, M. Garcia-Closas, L. El Ghormli, and N. Rothman, "Assessing the probability that a positive report is false: an approach for molecular epidemiology studies," Journal of the National Cancer Institute, vol. 96, no. 6, pp. 434-442, 2004. 